Crise(s) dans le monde ibérique et ibéro-américain

\title{
Crise économique et misère sociale dans Roble huacho (1947) de Daniel Belmar (Chili)
}

\section{Benoît Santini}

\section{(2) OpenEdition}

1 Journals

Édition électronique

URL : https://journals.openedition.org/cher/3537

DOI : 10.4000/cher.3537

ISSN : 2803-5992

Éditeur

Presses universitaires de Strasbourg

\section{Édition imprimée}

Date de publication : 1 décembre 2015

Pagination : 73-84

ISBN : 978-2-86820-913-9

ISSN : 1968-035X

\section{Référence électronique}

Benoît Santini, «Crise économique et misère sociale dans Roble huacho (1947) de Daniel Belmar (Chili) », reCHERches [En ligne], 15 | 2015, mis en ligne le 01 décembre 2021, consulté le 15 décembre 2021. URL : http://journals.openedition.org/cher/3537 ; DOI : https://doi.org/10.4000/cher.3537

\section{(c) (i) (8)}

Ce(tte) œuvre est mise à disposition selon les termes de la Licence Creative Commons Attribution Pas d'Utilisation Commerciale - Partage dans les Mêmes Conditions 4.0 International. 


\title{
Crise économique et misère sociale dans Roble huacho (1947) de Daniel Belmar (Chili)
}

\author{
Benoît SANTINI \\ Université du Littoral Côte d'Opale (Boulogne-sur-Mer) \\ Laboratoire HLLI
}

\begin{abstract}
A près une période de prospérité et de stabilité économique entre 1926 et 1929, le Chili subit de plein fouet la crise économique mondiale entre 1929 et 1932. Comme l'explique Luis Riveros, «la depresión económica vivida por Chile se derivó de la magnitud del efecto que la crisis internacional indujo en la balanza de pagos», et l'auteur souligne "el derrumbe de la economía chilena» à partir de 1931 (Riveros 2009: 1, 4). Roberto Toso et Álvaro Feller précisent: «El peor momento de la crisis se vivió durante 1932» (Toso-Feller 1983: 15). Le secteur minier, lié à l'exploitation du salpêtre, produit fortement touché depuis la fin de la Première Guerre mondiale, fut d'autant plus affecté par la crise, comme l’expose Sergio Villalobos: «la desocupación en la región salitrera alcanzó proporciones catastróficas y grandes grupos de obreros cesantes debieron ser trasladados al centro del país» (Villalobos 1983: 193). On assista à une chute de la production du cuivre, à une baisse de $33 \%$ des exportations et de $60 \%$ des importations, quant au PIB en 1937, il ne retrouva pas son niveau d'avantcrise (Sosnowski 1997: 293). Villalobos rappelle: "fue necesario que el Estado proporcionase alimentación diaria a la masa de desocupados», "tan grande fue la crisis que sus efectos se dejaron sentir por muchos años" (Villalobos 1983: 193). Ernesto Bohoslavsky, pour sa part, analyse la situation en ces termes:

En 1932 [...] Chile era el país más afectado en el mundo por la Depresión desatada dos años atrás. La caída en los precios de los productos primarios afectó gravemente a las exportaciones nacionales. [...] Aunque la crisis salitrera se venía arrastrando desde mediados de la década de 1920, estos años no hicieron sino profundizar el conflicto, arrojando a varios miles de mineros desempleados a los centros urbanos del país. En 1931 se agravó aun más la situación ya que cerraron más empresas dedicadas al cobre, al salitre y al carbón. [...] La Araucanía, región eminentemente agrícola, acusó recibo del galopante desempleo. Por su
\end{abstract}

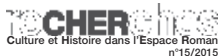


configuración minifundista de la tierra, Cautín era una de las provincias en las que había una proporción mayor de pequeñas propiedades agrarias familiares. La acentuada presencia de explotaciones familiares generó una rigidez del mercado de trabajo rural que dificultaba la potencial absorción de los parados (Bohoslavsky 2002: 547).

Ainsi, le Chili vit-il des années noires, et se retrouve-t-il confronté à une situation dramatique de misère et de chômage.

À cette époque (années 1920-1930), la création littéraire au Chili connaît un bel essor et la préoccupation sociale y occupe une large place. En poésie, Pablo Neruda, Gabriela Mistral ou Pablo de Rokha publient des œuvres essentielles de leur production ${ }^{1}$. Le dramaturge Antonio Acevedo Hernández publie en 1936 la pièce Chañarcillo, dont l'action se situe au cours de la première moitié du XIx ${ }^{e}$ siècle, en plein essor de l'exploitation du cuivre. En 1933 parait Llampo brujo de Sady Zañartu, roman qui aborde la question minière (Silva Castro 1960 : 65). C’est la "Generación de 1938 » plus particulièrement qui s’intéresse à la problématique sociale, comme en attestent les romans de Nicomedes Guzmán, Andrés Sabella ou encore Daniel Belmar. Cependant, peu de romans, pièces de théâtre ou poèmes se centrent sur la crise économique de l'époque en tant que telle, à la différence de la riche production nord-américaine traitant du sujet $^{2}$. Il faudra attendre 1947 pour que Daniel Belmar publie son roman Roble Huacho où il déploie diverses stratégies narratives afin d'évoquer les effets de la Grande Dépression au Chili entre 1929 et 1933. Peu de dates y sont précisées, mais le roman invite le lecteur à déployer toute son imagination et sa capacité à mettre en relation, à associer l'histoire ou les histoires narrées au contexte socioéconomique de ces années. Daniel Belmar (1906-1991), lauréat de nombreux

1 Rappelons l'engagement de P. Neruda, l'appartenance au communisme de P. de Rokha, la composition de poèmes sociaux par G. Mistral. Pablo Neruda publie en effet Residencia en la tierra en 1927 et España en el corazón en 1937, Gabriela Mistral Tala en 1938, Pablo de Rokha Canto de trinchera 1929-1933. N'oublions pas le recueil avant-gardiste de Vicente Huidobro, Altazor (1931), preuve de la vitalité créatrice dans le Chili de l'époque.

2 Consulter le document suivant donnant des précisions sur la littérature nordaméricaine et la crise de 1929: http://www.bpi.fr/modules/resources/download/ default/Decouvrir_la_Bpi/Documents/Bibliographies/ecrivains29.pdf

Comme l'écrit María Orozco Jesús Vera, «Entre los años 1935 y 1950 comenzaron a publicar novelistas que componen la generación de 1942, Generación Neorrealista o Generación de 1938, según las distintas denominaciones de la crítica. Jacobo Danke, Daniel Belmar, Nicasio Tangol, Gonzalo Drago, Coloane, Mariano Bahamonde, Oscar Castro, Reinado Lomboy, $\mathrm{M}^{\mathrm{a}}$ Carolina Geel y Fernando Alegría, entre otros, componen este grupo literario en el que se incluye también $\mathrm{M}^{\mathrm{a}}$ Luisa Bombal. Frente a la literatura criollista estos escritores proponen una estética que supere las limitaciones regionalistas y que contemple al hombre desde otra óptica. Su narrativa, inmersa en un profundo lirismo, se concreta en la búsqueda de la identidad del hombre, ese incesante bucear en las profundidades de la mente humana que le ofrecen las nuevas corrientes vanguardistas, especialmente el surrealismo" (Orozco Jesús Vera 1989: 39-40). 
prix littéraires, est chimiste et pharmacien de formation. La toile de fond de ses romans est très souvent la région de Concepción (VIIIe région, au nord de l'Araucanie). Roble huacho est le roman qui le fait connaître. Dans Coirón (1949), il se remémore son enfance à Neuquén, en Argentine. En 1955, il publie Los túneles morados, dont l'histoire se déroule à Concepción. Detrás de las máscaras, qui paraît en 1965, est écrit peu de temps après les cataclysmes des 21 et 22 mai 1960 qui détruisirent les villes du sud chilien et de Chiloé. Ainsi, ancre-t-il son écriture dans les régions du Chili qui deviennent le décor essentiel de ses romans.

Roble huacho, composé de seize chapitres, relate la misère d'un village du même nom situé dans la région de Cautín (IX ${ }^{\mathrm{e}}$ région, Araucanie). Son pharmacien Pancho Ríos, également narrateur homodiégétique en focalisation interne, brosse une série de portraits de villageois sans-le-sou, mais rapporte aussi des épisodes où il partage des moments complices avec ses amis et sa compagne, Solveig, rencontrée au cours du roman, une jeune fille mal mariée qui devra rejoindre de force son époux vivant à Puerto Saavedra (en Araucanie). Le roman nous amène à nous poser la question suivante: dans quelle mesure l'écriture narrative de Belmar dans Roble huacho ainsi que ses personnages sontils affectés par la dépression économique et comment ce roman parvient-il à «éclairer le concept de crise», pour reprendre les propos d'Edgar Morin (Morin 1976: 149)?

\section{Reflet de la crise économique dans Roble huacho}

La crise économique est le facteur principal de la déliquescence du village de Roble huacho, "poblacho» ou "pequeño pueblo de la antigua frontera mapuche» (Belmar 1947: 24), et le signifiant-clé du roman. Tout au long de l'œuvre, la récurrence lexicale du terme "crisis» ponctue et envahit l'énoncé, le dévastant: "¿Cuándo pasará esta maldita crisis?» (Belmar 1947: 18), se demande le narrateur, pharmacien de son état; il est fait allusion à «la más intensa de las crisis financieras que soportara el país» (Belmar 1947: 24) et le narrateur explique: «Hace ya mucho, antes que la crisis asomara su careta trágica sobre los campos ubérrimos, cada madrugada volcaba sobre el pueblo un desfile interminable de carretas fleteras», ou encore: «Es la crisis, y su angustia irremediable golpeando para nada» (Belmar 1947: 35, 37). Ce même narrateur ajoute, à propos de «El Guata $»^{3}$ Bascur, ferronnier: "Ahora, con la crisis se ha desbandado toda su clientela", "Hoy, la crisis ha transformado estas fondas en cantinas y prostíbulos clandestinos», "Los campesinos, atemorizados por la crisis, apenas sembraron este año lo indispensable para subsistir» (Belmar 1947 : 71, 86, 144). Il précise encore : «La crisis abruma el poblacho», «La crisis ha puesto en evidencia la fragilidad financiera de ciertos hacendados» (Belmar

3 En mapudungün, langue des Mapuches, «huata» signifie "estómago». «El Guata» Bascur «debe el sobrenombre al descomunal desarrollo de su panza» (Belmar 1947: 70). 
1947: 172) et évoque le destin d'un: «jornalero agrícola que, acorralado por la crisis en las secas lomas de Nuble 4 , bajara hacia el sur» (Belmar 1947: 198). Belmar multiplie les termes appartenant à la constellation sémantique de la crise: «fragilidad financiera», «deudas», «quiebras», «depresión» (Belmar 1947: 172-173). Ces allusions à la crise envahissent l'espace textuel comme la crise déferle sur le monde en 1929-1933. De façon insidieuse, celle-ci s'immisce progressivement dans le quotidien des habitants du village et dans certains secteurs-clé (secteur agricole), elle s'infiltre au fil des pages du roman et se propage telle une épidémie, confirmant ce quécrit Jean Baudrillard qui considère la crise comme une "catastrophe au ralenti» (Baudrillard 1990: 41).

La dépression économique crée un manque et un vide au sein de la population de Roble huacho, reproduction en miniature du Chili de l'époque. Aussi, soulignons-nous la portée signifiante des négations qui mettent en exergue ces carences: "Hace ya dos años que no me compro ni una camisa. [...] La gente no tiene ni para comer", "nadie compra nada. No hay dinero", «tampoco tengo nada. Ni siquiera un pollito que vender» (Belmar 1947: 18, 37, 42). Les négations deviennent l'un des traits distinctifs de l'écriture de Belmar. S’y associent en outre des termes évoquant l'absence: "privaciones, mínima cuantía, escasez de movimiento", "qué privaciones voy a tener que sufrir», «calle solitaria», «magros negocios» (Belmar 1947: 25, 28, 36). Lénonciation est ainsi rongée par les effets de la crise. Graphiquement, cette absence se manifeste aussi par les très nombreux points de suspension présents dans les conversations. Par exemple, lors d'une discussion entre Tito Andrade, petit employé de voirie, et «el rucio» Fernández, directeur de la succursale de la Caisse d'Epargne du village, ce dernier s’écrie: «No seas 'roto'... ¿de qué te quejas?... Tienes un buen sueldo... viáticos... y nadie a quien ayudar... En cambio yo... mi madre todo el tiempo enferma... y todavía estoy devolviendo el dinero que entregué a ese maricón desgraciado» (Belmar 1947: 27). Fernández fait référence au directeur de la Caisse d'Épargne de Pichi-Lleuque qui aurait malhonnêtement conservé une partie de la somme des «cheques fiscales» de la commune, remis par Tito, obligeant ce dernier à rembourser la différence. Les points de suspension abondent encore lorsque, après un flash-back narratif, alors que le narrateur lui conseille de s'adresser à Don Casimiro pour qu'il lui prête de l'argent, Tito lui répond: «Psss... Cien pesos a lo más... ¿sabes cuánto gano?... Apenas trescientos noventa pesos, con los descuentos... ¡Maricón desgraciado!... quizás qué privaciones voy a tener que sufrir por su culpa!...» (Belmar 1947: 27). Ces silences narratifs et vides verbaux confirment que «la littérature se définit [...] comme une herméneutique du silence» (Sartre 1972:29) et que "le silence est un moment du langage» (Sartre 1948: 32), «un acte verbal, un trou creusé dans le langage» (Louette 1995: 36). Si la crise ruine l'individu et mine son niveau de vie, elle semble creuser ses sillons dans le langage de certains personnages du roman, durement touchés par la précarité. Tito Andrade en vient d'ailleurs

4 Nuble se situe dans la région du Bío Bío, au nord de l'Araucanie. 
à sécrier, dans le chapitre I: «¿Qué año apocalíptico!»(Belmar 1947: 29). Nous avons affaire à une réécriture de l'Apocalypse biblique où s'active la «relation de coprésence» "entre deux textes», décrite par Gérard Genette (1982: 8), ici Le Nouveau Testament et Roble huacho, au service d'une dénonciation. Si les méfaits de Lucifer laissent la place au millénium, le village de Roble huacho, touché par la crise, la maladie et autres calamités, semble avoir atteint la fin d'une étape menant vers une re-naissance.

Face aux pauvres gens, apparaît une oligarchie opulente incarnée par Don Marcelo ( agente maderero») et Don Andrés ( «comerciante»). Ces derniers sont définis en ces termes: «Son poderosos». Don Nacho Cabrera, grand propriétaire, est désigné par son «estatura feudal» et «sus vastos dominios» (Belmar 1947: 50). L'aisance matérielle de Don Segundo est soulignée avec insistance: "Don Segundo es rico. Tiene fortuna" (Belmar 1947: 53). Ces insistances créent un contraste avec les carences des villageois de Roble huacho. Daniel Belmar, par l'implicite et l'habileté langagière, dénonce le pouvoir des groupes économiques et sociaux dominants si peu affectés par la crise.

Les habitants du village sont victimes de la misère et du chômage. Comme nous l'avons dit en introduction, le secteur minier - en particulier, l'exploitation du salpêtre - est particulièrement touché par la Grande Dépression. Ainsi, le narrateur fait-il allusion aux «cesantes de las salitreras»(Belmar 1947: 37), reconvertis en cantonniers à cause de la crise. Il évoque les déplacements et les mutations provoqués par celle-ci: «La semana pasada se fue Tito Andrade, trasladado al Departamento de Caminos de la provincia de Malloco», la succursale de la Caisse d'Épargne ayant en effet fermé "por escasez de movimiento» (Belmar 1947: 194). Est également mentionnée la précarité des logements d'une frange de la population qui vit dans des bidonvilles:

[...] se alza un ruinoso tendido de informes viviendas, terrosas, obscuras, miserables. Con ligeras variantes, todas son iguales: sin ventanas, los muros roídos por el tiempo, de podridas maderas desajustadas formando anchas grietas por donde se cuela el frío y la lluvia, el viento y el polvo, el invierno y el desamparo (Belmar 1947: 37).

Le narrateur adopte un discours narratif fondé sur l'esthétique de la disparition et du vide: les énumérations, les asyndètes, les rythmes binaires et ternaires se multiplient. L'énonciation hachée, entrecoupée de virgules, agressée par de nombreuses occlusives ("podridas», "polvo», "desamparo») et des allitérations violentes («tendido», «terrosas», «informes», «formando», «frío») est à l'image de la crise qui ravage populations et habitations et dévore le village qui semble se réduire comme une peau de chagrin, anéanti par les circonstances économiques. La description de la maison du vendeur de chaussures, Vivanco, le confirme: «las vigas desnudas, ahumadas, muestran colgantes cendales de telarañas cuajadas de esqueletos de moscas [...]. Las hojas de periódicos y de revistas con que han empapelado los muros se ven rotas", "Aquí y allá, míseros muebles» (Belmar 1947: 64). Les lieux de vie s'effritent et se décomposent, tout comme leurs habitants. 


\section{Roble huacho, village anéanti}

À la misère, s'ajoutent les maladies. Les descriptions d'infirmités, d'affections ou d'autres blessures contribuent à créer une atmosphère de destruction. Les motifs narratifs de la misère, des maladies et de la laideur se combinent dans une description très zolienne. Ainsi, la population pauvre des faubourgs du village est-elle décrite comme: "una población horrenda, una sociedad de harapos y de hambre, piojosa, comida por la sarna y la miseria» (Belmar 1947: 37). La rage ou encore l'épilepsie affectent certains des personnages, par exemple le fils de la lavandière, Juan Manuel (Belmar 1947: 134) ou encore le fils de la villageoise Margarita, ausculté par Pancho dont le verdict est sans appel: «Es sarna, la vulgar, molesta sarna, que martiriza las noches de los pobres en el mundo» (Belmar 1947: 68). La dégradation est présentée comme un legs et les enfants de ces familles misérables sont décrits en ces termes: "la chiquillería gritona, siempre famélica», "casposas piernas de alambre y gruesas rodillas raquíticas» (Belmar 1947: 38). La maigreur et la faim népargnent ni les enfants ni leurs géniteurs et certaines des descriptions rappellent des tableaux du Siècle d'Or espagnol (Murillo, José de Ribera) et les "peintures noires» de Goya ${ }^{5}$. Le pharmacien Pancho est donc souvent sollicité par les villageois afin qu'il ausculte certains de leurs proches. En raison de la crise économique, le village est devenu un véritable désert médical où le pharmacien fait office de médecin: "Cuándo será el día en que se venga un médico a este pueblo desgraciado... Estoy transgrediendo la ley... No debo atender enfermos... Pero esta gente tampoco se puede morir así» (Belmar 1947: 66). Pancho, protagoniste et narrateur, se centre sur les descriptions médicales, lesquelles deviennent un procédé scriptural essentiel. La conséquence ultime de la crise est le décès, comme dans le cas du nourrisson dont Pancho raconte la venue au monde: "[...] y de pronto, como un vómito de sangre y de inmundicia, aparece una criatura sobre el tibio pellejo que lo espera» (Belmar 1947: 46). La monstruosité de la description semble ne laisser aucune issue aux populations touchées par la crise. Même la naissance est marquée par la destruction.

Les esthétiques de la laideur et de la décomposition deviennent des procédés scripturaux essentiels dans le roman. Les descriptions du narrateur, fortement expressives, intensifient les effets destructeurs de la crise. Avec une précision et une rigueur scientifiques, Pancho Ríos fait un portrait de la laideur, qui illustre bien l'analyse de R. Polin: "Ce qui est laid offusque, non pour ce qu'il n'a pas, mais pour ce qu'il a. Il est vraiment, non point absence de réalité, mais présence de laideur, non pas manque, mais trop plein» (Polin 1966: 260). Les descriptions de monstruosités et de corps difformes remplissent l'espace textuel: ainsi, le chef

5 Le traitement scriptural de la maladie est récurrent dans les années 1940 dans la littérature occidentale, comme dans La peste de Camus (1947). On retrouve également un intérêt pour l'écriture de la maladie physique et mentale mais surtout au XIX ${ }^{e}$ siècle dans la littérature française (Zola, Flaubert, Baudelaire) ainsi que dans l'Antiquité (Hérodote). 
de gare qui corrompt la jeune Armanda en lui proposant des rapports sexuels en échange de ses «hermosos billetes» se caractérise par ses: «manos horribles, feas cicatrices» (Belmar 1947: 44). Le vice semble donc se manifester également par le physique repoussant de cet homme. Quant à la fille du vendeur de chaussures, Vivanco, elle est atteinte de gonorrhée et Pancho décrit en détail le sexe infecté:

[...] un sexo en toda su cruda realidad, un sexo torcido, de pelos espesos y crespos; un sexo semejante a una boca deforma, deshumanizada, como la de algún horrible animal desconocido; una boca monstruosa, macerada, de labios negros; uno de ellos atrozmente hinchado, tumefacto, doloroso. De entre ellos fluye como un río lento y asqueroso, gruesa secreción lechosa y nauseabunda (Belmar 1947 : 65-66).

L'énumération, l'absence de verbes, l'abondance de virgules et les parallélismes de construction décomposent la syntaxe, reproduisant ainsi la déliquescence corporelle de la jeune fille. Cette esthétique de la déformation est perceptible aussi dans la description du fils de la lavandière Margarita: "deforme cabezota » (Belmar 1947: 67), "le ha aparecido una 'flota' de granos en todo el cuerpo", «repugnante», «rojas excoriaciones», «sarna» (Belmar 1947: 68). Tous les sens du lecteur sont sollicités pour partager avec le narrateur son dégoût mais aussi sa compassion envers les malheurs de cette population miséreuse. L'indien Cayupi, atteint de tuberculose, est décrit comme suit: «una tos seca, dolorosa, sacude a ratos, como una brizna, al indio mísero", "rostro consumido por la enfermedad, devorado por la fiebre» (Belmar 1947: 69). Enfin, le Guata Bascur apparait distordu dans une description grotesque: «descomunal desarrollo de su panza», "es un paquidermo lento y pesado, gigantesco, de patas tiesas por el reumatismo»(Belmar 1947: 70). La crise qui dégrade le monde semble se matérialiser et s'engouffrer dans les corps des individus, les décomposant à leur tour. Le langage est lui aussi déformé par cette même crise, comme le démontrent les nombreux chocs phoniques ( $k$, ch, rr) (Belmar 1947: 36), reflets du pourrissement qui atteint désormais l'espace de l'action: «Las rudas carretas fleteras de otro tiempo han desaparecido. Tal vez pudren sus maderas [...] al lado de las 'canchas' atiborradas de troncos derribados y cuarteados, hundidos, socavados [...], desmenuzados» (Belmar 1947: 36).

Tout le village est plongé dans la pénombre comme nous le constatons à plusieurs reprises, le mot "obscuridad» et ses dérivés revenant souvent: "la obscura silueta del convento franciscano", "el obscuro fondo de la noche" (Belmar 1947: 15, 19). L'espace géographique, mais aussi le silence et le souvenir sont marqués du sceau de l'obscurité, y compris les traits physiques de certains habitants, en particulier les yeux et la chevelure noirs. Michel Pastoureau rappelle que l'utilisation de la couleur est «culturelle et idéologique» et renvoie à des «enjeux économiques, politiques, sociaux, symboliques s'inscrivant dans un contexte précis» (Pastoureau 2008: 12).

Le texte semble s'auto-détruire par le biais de ces procédés discursifs qui font de la bourgade de Roble huacho un espace en pleine désagrégation. Roble huacho devient le village de tous les vices et des péchés capitaux. 
Paul Ricœur, qui étudie le concept de crise dans son acception large, l'analyse ainsi: "percevoir une situation comme crise, dirai-je dans la ligne de Landsberg et de Mounier, c'est ne plus savoir quelle est ma place dans l'univers, ne plus savoir quelle hiérarchie stable de valeurs peut guider mes préférences» (Ricœur 1988: 1-19). Dans le roman, effectivement, les valeurs sont en perdition et certains personnages en perte de repères en sont l'illustration. Lévocation de la luxure est par exemple récurrente. Les personnages se rendent dans les bas-fonds, à savoir la maison close tenue par Doña Eva et le contact des corps est suggéré par des allusions suffisamment explicites: «Siento junto a mi mejilla el contacto ardiente de un rostro febril y humedecido», "Aliro García [...] palmotea suavemente las gruesas nalgas que no dejan de temblar» (Belmar 1947: 22, 23). Sebastián Elgueta a une relation avec une mineure et s'en enorgueillit: «Estaba re buena... Se abrió como un lienzo » (Belmar 1947: 96-97). Cette référence érotique et l'obsession du désir charnel créent une deuxième crise au sein du roman: une crise familiale, car la tante de la Lucha, séduite par Elgueta, fait appel aux carabiniers afin qu'ils rétablissent l'ordre. Le paroxysme de la luxure ou de la perversion sexuelle est atteint lorsque «el rucio» Fernández raconte un cas de zoophilie: celui de Patas de Huasca copulant avec des poules (Belmar 1947: 31-32). En sus de la luxure et du péché capital, apparaissent divers exemples de vices comme le vol, lorsque Óscar Vegas, "tesorero comunal», puise dans les caisses du village (Belmar 1947: 52), ou l'alcoolisme, traditionnellement considéré tantôt comme vice, tantôt comme maladie et qui concerne bon nombre de personnages du roman: «el vino vuelve a llenar las copas» "Estoy borracho» (Belmar 1947: 56, 107). Ainsi, se produit-il un véritable bouleversement des valeurs, une crise morale qui découle tout naturellement de la crise économique, tout en étant en même temps un peu la cause de celle-ci, mais aussi de la Seconde Guerre mondiale, qui vient de prendre fin lors de la publication et donc non encore survenue dans l'histoire narrée. Le village est frappé par une malédiction, une sorte de châtiment divin comme le suggère le narrateur, lequel évoque les «dioses implacables» (Belmar 1947: 36) qui déterminent le destin tragique de Roble huacho. À travers la réécriture de la chute de Troie (dont les raisons économiques ont été transformées en raisons héroïques dans les poèmes de l'Antiquité), chute chantée dans l'Iliade, l'Odyssée et l'Enéide et dont les dieux sont en grande partie responsables, Roble huacho devient en quelque sorte, par l'implicite, un village mythifié, une sorte d'antichambre du Paradis, un Purgatoire, un lieu de passage obligé avant la survenue d'une époque meilleure.

\section{Récupérer une identité, rétablir la justice: résister face à la crise}

Pour mieux lutter contre la crise, les personnages réaffirment l'identité chilienne. Lanalyse d'Iván Carrasco peut permettre d'éclairer cette question de l'identité dans le roman de Belmar:

En el discurso literario estas identidades son representadas, configuradas o proyectadas principalmente en la actuación, caracterización y diálogo de personajes 
o tipos humanos representativos, en determinados enunciados ideológicos o valorativos de los sujetos enunciativos y en los discursos metatextuales complementarios del autor (Carrasco 2011:23-32).

C'est bien par le biais du langage que les protagonistes revendiquent leur identité. Il devient un instrument de cohésion sociale et une arme contre la décomposition de la société en proie à la crise. Les surnoms populaires, courants au Chili, donnés aux personnages reproduisent la familiarité conversationnelle: «el rucio» («blond»), «la Lucha», «Nacho Cabrera» (Belmar 1947: 145). Ce sont aussi des termes renvoyant aux traditions et à l'alimentation chiliennes: «el mote», boisson à base de grains de blé, «la once», sorte de goûter pratiqué au Chili, "la empanada», chausson à la viande, "la cazuela» et "el pebre», condiment, "la coliza», pain chilien, "los digüeñes», champignons, "los pequenes », petits chaussons à la viande (Belmar 1947: 40, 117, 85, 133, 106, 215). Les «chilenismes» (et parfois les termes d’origine mapuche) abondent: «está re buena", "al tiro», "esta cabra» (ibid., 102). Ils permettent aux personnages de se recentrer sur l'essentiel, de reconstruire un village voire un pays à partir de ce qui leur est le plus propre. La nourriture est lien social et, comme l'explique Barthes, "un véritable signe, c'est-à-dire peut-être l'unité fonctionnelle d'une structure de communication; [...] c'est toute la nourriture qui sert de signe entre les participants d'une population donnée» (Barthes 1961: 980). Le signe est, dans le cas qui nous intéresse, la conscience d'appartenir à une Histoire et à un pays, de partager une identité par l'alimentation (ce qui est valable aussi pour le langage), de s'unir en période de crise destructrice.

Le narrateur charpente également son discours narratif et l'espace textuel du roman en construisant une architecture du village : ainsi, évoque-t-il la «botica», la «plaza», l'«Hotel». Si le village est un lieu parfois infernal, les nombreuses descriptions d'un Paradis terrestre et d'un locus amoenus introduisent un havre de paix: «el paisaje se me entra por los ojos hasta el corazón», "el río es una vena abierta, palpitante, por donde fluye el agua viva y eterna " (Belmar 1947: 132-133), "un lujurioso oleaje de verdura sobre los valles», " una tierra convertida en flor» (Belmar 1947: 142). Ce débordement de vie et de couleurs surpasse la pénombre et la décomposition du village et de ses habitants. En outre, l'apparente structure fragmentaire de la narration se reconstruit à travers le rôle prépondérant de Pancho dans le roman. La riche galerie de personnages crée l'impression d'une explosion des sujets narratifs dont les expériences convergent cependant vers la figure de Pancho Ríos: le pharmacien-«médecin» est sollicité sans cesse par les villageois. Ses prescriptions médicales apaisent les «crises» de douleur de ses patients, mais il sait aussi, de façon symbolique, trouver des remèdes à une autre crise, la crise économique. Il redonne la santé à ceux qui lui rendent visite, c'est-à-dire à ceux qui sont les plus touchés par la dépression économique. Lincendie final de la pharmacie est d'autant plus symbolique: «Esto es el fin. Y el comienzo» (Belmar 1947: 221). Tel le Phénix, la pharmacie part en fumée, mais le feu purificateur et le cataclysme semblent annoncer une 
régénération pour le village désormais libéré de ses maux, et n’ayant plus besoin d’ordonnance médicale.

Pancho Ríos, enfin, se caractérise par son humanité et sa générosité, il donne de son temps aux villageois et refuse toute rémunération pour ses consultations (Belmar 1947: 67, 68). Il est un peu le fil qui maintient le village en vie et défend les pauvres gensqui le peuplent. Lorsqu'il est convoqué par la justice suite à l'inhumation du nouveau-né près d'une habitation, acte considéré comme illégal (Belmar 1947: 79, 83), il apparaît comme un nouveau Bon Samaritain: «Es una inhumación ilegal, por ignorancia. Me parece el colmo del rigor la detención de estas pobres mujeres por algo que no alcanza a ser un hecho delictuoso» (Belmar 1947 : 83). Pancho rétablit une justice, s'emploie à redonner la santé aux malades et affronte avec courage la crise et le monde sans pitié de la Justice.

\section{Conclusion}

Roble huacho apparaît comme un roman de résistance, une réaction face à la crise des années 1929-1933. Le titre du roman, qui est le nom du village où se déroule l'action, est symbolique:

En la misma esquina de la plaza, [...] un árbol aislado eleva hacia la altura su tronco centenario. El espeso y frondoso follaje es una copa de pájaros, un nidal de trinos y de alas, una guitarra verde y rumorosa en los dedos del viento.

Es un roble solitario, un roble 'huacho'. Ha entregado su nombre al pueblo que parece cobijarse bajo su sombra (Belmar 1947: 133).

Le chêne, unique arbre du village, est traditionnellement l'arbre de la justice. Inébranlable, il résiste aux assauts de la crise et de la misère, tout en conservant sa vitalité, comme le démontrent les termes «elevarse», "frondoso follaje», «trinos», «guitarra verde». Cet arbre solitaire, empreint de dynamisme, n’annoncerait-il pas une lueur d'espoir, le rétablissement d'une justice et la fin des souffrances des villageois?

Ce roman, reflet d'une époque marquée par la Grande Dépression et les deux guerres mondiales, restructure un univers dominé par le chaos. Le lyrisme de certaines évocations, l'histoire d'amour vécue par Pancho et la jeune Solveig, l'espace naturel où se réfugie le narrateur, son sens de la justice et sa générosité soulagent le discours narratif tourmenté. À travers la dénonciation des abus de la justice corrompue, représentée par le juge Walter (Belmar 1947: 75, 81), des grands propriétaires, représentés par Don Nacho Cabrera, le narrateur belmarien montre du doigt les méfaits du capitalisme et la corruption (Belmar 1947: 150, 154), la brutalité des oligarchies capables d'utiliser la violence pour faire taire les protestataires et leurs revendications. C'est le cas de Nacho Cabrera qui s'en prend au viejo Anguita: "La pesada argolla de la 'penca' cae arteramente sobre la frente venerable que se quiebra con un chasquido trágico» (Belmar 1947: 150). C'est que ce dernier a porté plainte contre le propriétaire au sujet de limites de terrains: «La demanda contra don 'Nacho' Cabrera fue acogida favorablemente 
en el juzgado de Temuco; el informe del agrimensor que vino a rectificar las líneas, influyó decisivamente en su favor pese a un intento de soborno por parte del rico» (Belmar 1947: 145). Cette brutalité verbale et physique trouve un écho dans la violence de la crise incarnée par ces hommes puissants qui en deviennent l'allégorie. Le roman "éclaire» donc bien «le concept de crise» dans son acception large: crise des valeurs, crise économique, crise narrative (fragmentation textuelle) et, à travers le prisme de son héros et narrateur, Pancho Ríos, Roble huacho démontre que la solidarité et l'altruisme sont un premier pas vers la résolution de toute crise.

\section{Bibliographie}

Barthes, R., 1961, «Pour une psycho-sociologie de l'alimentation contemporaine », Annales. Économies, Sociétés, Civilisations, n 5, p. 977-986.

Baudrillard, J., 1990, La Transparence du mal. Essai sur les phénomènes extrêmes, Paris, Galilée.

Belmar, D., 1947, Roble Huacho, Santiago du Chili, Cultura.

Bohoslavsky, E., 2002, "Desempleo, organización y política. Los trabajadores rurales del sur chileno frente a la Gran Depresión», Anuario de estudios americanos, Sevilla, vol. 59, no 2, p. 541-563.

Carrasco, I., «Identidades en la poesía canónica de Gabriela Mistral», Estudios filológicos, Valdivia, 48 (nov. 2011), p. 23-32.

Genette, G., 1982, Palimpsestes. La littérature au second degré, Éditions du Seuil, Points/ essais.

Louette, J.-F., 1995, Silences de Sartre, Presses Universitaires du Mirail, Essais de littérature, Cribles.

Morin, E., 1976, «Pour une crisologie», Communications, n 25, p. 149-163.

Orozco Jesús Vera M., "La narrativa de María Luisa Bombal: principales claves temáticas», Cauce, Revista de Filología y su Didáctica, n 12, 1989, p. 39-56.

Polin, R., 1966, «Rien n'est beau que le laid... ou presque », Revue d'esthétique, p. 258-265.

Pastoureau, M., 2008, Noir: histoire d'une couleur, Paris, Éditions du Seuil.

Ricœur, P., 1988, «La crise: un phénom Ricœur ène spécifiquement moderne?», Revue de théologie et de philosophie, 120, p. 1-19.

Riveros, L., 2009, «La gran depresión (1929-1932) en Chile», Facultad de Economía y negocios, Universidad de Chile, Departamento de Economía, Serie "Documentos de Trabajo", p. 1-25.

Sartre, J.-P., 1948, Qu'est-ce que la littérature?, Paris, Gallimard, Idées.

Sartre, J.-P., 1972, L'idiot de la famille, Paris, Gallimard.

Silva Castro, R., 1960, Evolución de las Letras chilenas. 1810-1960, Santiago, Editorial Andrés Bello. 
Sosnowski, S., 1997, Lectura crítica de la literatura americana. Vanguardias y toma de posesión, Caracas, Biblioteca Ayacucho.

Toso, R., Feller, Á., 1983, «La crisis económica de la década del treinta en Chile: lecciones de una experiencia», Santiago, Serie de Estudios Económicos, Documentos de investigación, $\mathrm{n}^{\circ}$ 21, p. 7-56.

Villalobos, S., 1983, Breve Historia de Chile, Santiago de Chile, Editorial Universitaria. 